\title{
Magnetic fluctuations and superconductivity in $\mathrm{YbPd}_{2} \mathrm{Sn}$
}

\author{
A. Amato ${ }^{a}$, B. Roessli ${ }^{b}$, P. Fischer ${ }^{b}$, N. Bernhoeft ${ }^{c}$, \\ A. Stunault ${ }^{d}$, C. Baines ${ }^{\text {a }}$, A. Dönni ${ }^{\text {e }}$ and H. Sugawara ${ }^{f}$ \\ ${ }^{\mathrm{a}}$ Lab. for Muon-Spin Spectroscopy, Paul Scherrer Institute, CH-5232 Villigen PSI, \\ Switzerland \\ ${ }^{\mathrm{b}}$ Lab. for Neutron Scattering, Paul Scherrer Institute 83 ETH Zurich, CH-5232 \\ Villigen PSI, Switzerland \\ ${ }^{\mathrm{c}}$ CEA-Grenoble, Av. des Martyrs, F-38054 Grenoble cedex, France \\ ${ }^{\mathrm{d}}$ Institut Laue-Langevin, Av. des Martyrs, F-38054 Grenoble cedex, France

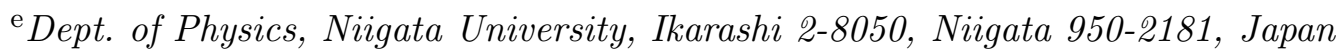 \\ ${ }^{\mathrm{f}}$ Dept. of Physics, Tokyo Metropolitan Univ., Minami-Ohsawa 1-1, Hachioji-Shi, \\ Tokyo 192-0397, Japan
}

\begin{abstract}
We report muon spin relaxation and inelastic neutron measurements on the Heusler system $\mathrm{YbPd}_{2} \mathrm{Sn}$. Localised anisotropic and quasi-elastic $\mathrm{Yb}$ magnetic fluctuations are observed below $T=150 \mathrm{~K}$. Both $\mu \mathrm{SR}$ and neutron data indicate a slowing-down of the spin-fluctuations, upon lowering the temperature, similar to that observed in Kondo lattices. The temperature dependence of the quasi-elastic neutron signal is compatible with a crystal-electric field scheme having a $\Gamma_{7}^{\mathrm{CEF}}$ ground state. The muon depolarization rate exhibits an additional contribution upon decreasing the temperature below $T_{\mathrm{c}}$ suggesting a close interplay between magnetic fluctuations and the superconducting state.
\end{abstract}

Key words: $\mu \mathrm{SR}$ spectroscopy, inelastic neutron scattering, superconductivity, Kondo effect

Corresponding author:

Alex Amato, Paul Scherrer Institute, CH-5232 Villigen PSI, Switzerland

Phone : +41-56-310 32 32; Fax : +41-56-310 32 94; Email : alex.amato@psi.ch 
The interplay between magnetism and superconductivity (SC) has been attracting interest in view of the possible rôle played by the magnetic fluctuations in the pairing mechanism between electrons leading, for example, to the formation of Cooper pairs with non-zero orbital moment [1]. In this context much effort is presently devoted either to systems which are close to a quantum critical point, i.e. where the superconducting state appears to be favored by the presence of critical spin-fluctuations, or to the so-called heavy-fermion compounds where the strong hybridization between the $f$ and conduction electrons appears to be the necessary ingredient for the occurrence of unconventional SC. On the other hand, rare-earth based systems like $\mathrm{RRh}_{4} \mathrm{~B}_{4}$, Chevrel phases and $\mathrm{RNi}_{2} \mathrm{~B}_{2} \mathrm{C}$ (where $\mathrm{R}$ represents the rare-earth ion) may be modelled in terms of spatially isolated electron subsystems, where the $4 f$ magnetic moments interact only weakly with the superconducting electrons. In this light, the Heusler system $\mathrm{YbPd}_{2} \mathrm{Sn}$ plays a particular rôle, since on one side the proportion of magnetic $f$-ions is high and on the other side the nearest-neighbor distance between rare-earth ions is rather short $(\sim 4.5 \AA)$ preventing a clear isolation between the magnetic and superconducting sublattices $[2,3]$.

$\mathrm{YbPd}_{2} \mathrm{Sn}$ exhibits coexistence of $\mathrm{SC}\left(T_{\mathrm{c}}=2.3 \mathrm{~K}\right)$ and simple antiferromagnetism $\left(T_{\mathrm{N}}=0.28 \mathrm{~K}\right)$ characterized by a propagation vector $\mathbf{k}=(001)$ and magnetic moments aligned along the [111] direction [4]. The value of the ordered magnetic $\mathrm{Yb}$ moments $\left(1.4 \mu_{\mathrm{B}}\right)$ is slightly smaller than the value $1.71 \mu_{\mathrm{B}}$ expected from the crystal-field ground state doublet $\Gamma_{7}^{\mathrm{CEF}}$. Starting from the parent non-magnetic and superconducting system $\mathrm{YPd}_{2} \mathrm{Sn}\left(T_{\mathrm{c}}=4.55 \mathrm{~K}\right)$, it was shown that the depression of $T_{\mathrm{c}}$ in $\mathrm{YbPd}_{2} \mathrm{Sn}$ is characterized by an anomalously large value of the product $N(0) J_{\text {sf }}^{2}$, where $N(0)$ is the density of states at the Fermi level per atom per spin direction and $J_{\text {sf }}$ represents the exchange interaction between the conduction electron spins and the localized $\mathrm{Yb} 4 f$ spin [5]. This points towards a strong hybridization and Kondo-type resonant scattering between localized and conduction electrons in this system.

In this light, to gain insight into their possible rôle on the superconducting state, it is of interest to characterize the thermal evolution of the spinfluctuation spectrum at low temperatures. In this paper we present muon-spin relaxation and inelastic neutron scattering (INS) measurements performed at the Paul Scherrer Institute (surface muons instruments GPS and LTF; coldneutron three-axis spectrometer TASP) and at the Institut Laue-Langevin (cold-neutron three-axis spectrometer IN14). For all measurements, we used polycrystalline samples from the same batch which was previously investigated at low temperature by neutron diffraction [4].

The $\mu \mathrm{SR}$ measurements were performed with conventional zero-field (ZF) and longitudinal-field (LF) techniques [6] using either a continuous ${ }^{4} \mathrm{He}$-cryostat (GPS, base temperature $1.7 \mathrm{~K}$ ) or a dilution ${ }^{3} \mathrm{He}-{ }^{4} \mathrm{He}$ refrigerator (LTF, base temperature $\sim 30 \mathrm{mK})$. For both instruments the same sample was investi- 
gated (powder glued on a high purity sample silver holder by mixing it with less than $2 \% \mathrm{GE}$ varnish). The neutron measurements were made at fixed final wavevector with the sample enclosed in an Al-container in a ${ }^{4} \mathrm{He}$ cryostat of ILL-type which attains the base temperature of $T=1.5 \mathrm{~K}$. In order to characterize the inelastic signal, the measurements had to be performed with different configurations yielding energy resolutions at elastic positions between $\sim 50 \mu \mathrm{eV}$ and $\sim 0.8 \mathrm{meV}$. In all cases a filter (cold Be or pyrolitic graphite) was installed in the beam to avoid contamination of the signal by higher-order wavelengths.

Whereas for temperatures above $50 \mathrm{~K}$, the ZF $\mu \mathrm{SR}$ signal is described by a single exponential function, at lower temperature it is best fitted assuming the depolarization function

$$
P_{\mu}(t)=A_{\text {slow }} \exp \left(-\lambda_{\text {slow }} t\right)+A_{\text {fast }} \exp \left(-\lambda_{\text {fast }} t\right)
$$

Below $T=50 \mathrm{~K}$, both the ratio between the amplitudes and the depolarization rate $\lambda_{\text {slow }}$ are found to be temperature independent with $A_{\text {slow }} / A_{\text {fast }}=0.50(5)$ and $\lambda_{\text {slow }} \sim 0.55(3) \mathrm{MHz}$, whilst $\lambda_{\text {fast }}$ varies as shown in Fig. 1 . The exponential character of the muon polarization decay, as well as the observation of a similar signal in LF measurements $\left(H_{\text {ext }}=2 \mathrm{kOe}\right)$ performed above $T_{\mathrm{c}}$, reveal the existence of fast $\mathrm{Yb}$ spin fluctuations measurable within the $\mu \mathrm{SR}$ timewindow. In addition, the presence of a two-component depolarization function of the observed amplitude ratio is indicative of a strong spatial anisotropy of such spin fluctuations producing an anisotropic field distribution at the muon site (i.e. $M_{2, \|} \gg M_{2, \perp}$ where $M_{2, i}$ represents the second moment of the static field distribution at the muon site along different directions). The portion of the muon ensemble possessing an initial polarization along the principal direction of the field distribution at the muon site is reflected by the first component of Eq. 1, with

$$
\lambda_{\text {slow }}=\tau \gamma_{\mu}^{2} 2 M_{2, \perp}
$$

where $\gamma_{\mu}$ is the gyro-magnetic ratio of the muon and $\tau$ represents the characteristic time of the anisotropic Yb-spin fluctuations. The second component of Eq. 1 is dominated by the other spin directions and its depolarization rate can be expressed as

$$
\lambda_{\text {fast }}=\tau \gamma_{\mu}^{2}\left(M_{2, \|}+M_{2, \perp}\right) \simeq \tau \gamma_{\mu}^{2} M_{2, \|}
$$

Additional information on the spin-fluctuations is obtained from inelastic neutron measurements for which the cross section reflects the imaginary part of the wave-vector and frequency dependent susceptibility $\chi^{\prime \prime}(\mathbf{q}, \omega)$. In a single 
ion approximation, crystal electric field (CEF) transitions yield a q-independent susceptibility:

$$
\frac{\chi^{\prime \prime}(\omega)}{\pi \omega}=\sum_{m} \chi_{\mathrm{C}}^{m} P_{m m}(\omega)+\frac{1}{2} \sum_{m \neq n} \chi_{\mathrm{vv}}^{n m}\left[1-\exp \left(-\beta \Delta_{n m}\right)\right] P_{n m}\left(\omega-\Delta_{n m}\right)(4)
$$

where the sums go over the CEF-levels and $\chi_{\mathrm{C}}$ and $\chi_{\mathrm{vv}}$ are the Curie and Van Vleck susceptibilities, respectively [7]. When dominated by single relaxation rate decay of the magnetisation, the scattering function $P_{n m}(\omega)$ reads

$$
P_{n m}(\omega)=\frac{1}{\pi} \frac{\Gamma_{n m}(T)}{\omega^{2}+\Gamma_{n m}^{2}(T)}
$$

High-resolution inelastic neutron measurements in $\mathrm{YbPd}_{2} \mathrm{Sn}$ have indeed revealed a quasi-elastic signal characteristic of localized excitations arising from single-site fluctuations with the energy dispersion of the form of Eq. 5. An effective CEF scheme can be inferred from the temperature dependence of the frequency integrated weight of the quasi-elastic signal. In Fig. 2 the best fit to the experimental data is given. The fit yields the cubic CEF parameters $W=-6.629 \mathrm{~K}$ and $x=-0.740$ which are close from the values reported by Li et al. [9]. With these parameters, the excited $\Gamma_{8}^{\mathrm{CEF}}$ state lies $4 \mathrm{meV}$ above the $\Gamma_{7}^{\mathrm{CEF}}$ ground state (see inset Fig. 2) which is in reasonable agreement with our INS data which reveal a transition at $3.6 \mathrm{meV}$ (not shown).

In the absence of a $\mathbf{q}$-dependence of the $\mathrm{Yb}$-fluctuations, the local probe $\mu \mathrm{SR}$ data can directly be connected to the dynamical susceptibility since

$$
\tau=\frac{1}{\Gamma_{\mu \mathrm{SR}}}=\frac{1}{\chi_{\mathrm{C}}} \lim _{\omega \rightarrow 0} \frac{\chi^{\prime \prime}(\omega)}{\pi \omega} .
$$

For a single ion in the presence of Kondo coupling, the dynamical susceptibility has been obtained within the NCA approximation by Cox et al. [8]. At very low temperature (i.e. below the characteristic Kondo temperature), the dynamical susceptibility assumes a single Lorentzian form with a constant dynamical width, whereas a $T^{\beta}$-like temperature dependence of this latter with $\beta=$ $1 / 2$ is expected at high temperature when the full degeneracy of the Yb-spin configuration is recovered. The experimental thermal variation of $\Gamma_{\mu \mathrm{SR}} \propto \lambda_{\text {fast }}^{-1}$ and of the width of the quasi-elastic Lorentzian neutron signal do indeed show a constant value at low temperatures just above $T_{\mathrm{c}}$. On the other hand the temperature dependence at high temperatures suggests an exponent $\beta$ value much higher than $1 / 2$. Such a behavior could reflect the presence of CEF effects and/or muon diffusion effects acting on the observed muon depolarization at high temperatures. If on one hand the importance of CEF effects is confirmed by the level scheme extracted from our INS measurements (see Fig. 2), on the 
other hand the presence of muon diffusion at high temperatures, which could mask the effects of the Yb-fluctuations on $\lambda_{\text {fast }}$, is suggested by the observation of a simple exponential depolarization. Assuming muons not to diffuse at high temperatures, an initial Gaussian depolarization should have been observed, reflecting the expected Gaussian field distribution at the muon site caused by the nuclear moments of the ${ }^{171,173} \mathrm{Yb},{ }^{105} \mathrm{Pd}$ and ${ }^{115,117,118} \mathrm{Sn}$ isotopes [11]. The observed exponential decay of the polarization demonstrates the mobility of the muon at high temperature leading to motional narrowing phenomena masking the intrinsic effects of the $\mathrm{Yb}$-fluctuations on the field distribution at the muon site.

As shown in Fig. 1, the $\mu$ SR depolarization rate $\lambda_{\text {fast }}$ exhibits a clear increase upon decreasing the temperature below $T_{\mathrm{c}}$. The relation between the observed increase and the presence of the superconducting state is confirmed by measurements performed in the LF configuration for temperatures below $T_{\mathrm{c}}$ as shown in the inset of Fig. 1. These measurements performed at $1.8 \mathrm{~K}$ in fieldcooling procedures indicate that the additional increase of the depolarization rate occurring below $T_{\mathrm{c}}$ is canceled when the external magnetic field is higher than the critical field $H_{c 2}(T=1.8 \mathrm{~K}) \simeq 250 \mathrm{G}$ [12].

In the following we discuss different possible origins for the increase of the $\mu \mathrm{SR}$ depolarization rate in the superconducting state. An obvious origin is related to the formation of the superconducting gap which decreases the number of available conduction electrons capable to participate to a Kondo interaction with the localized $4 f$-spin. However, the reduction of the ordered magnetic moment, below $T_{\mathrm{N}}$ determined by neutron scattering experiments [4], compared to the calculated value expected from the $\Gamma_{7}^{\mathrm{CEF}}$ ground state, suggests that the Kondo interaction is still effective much below $T_{\mathrm{c}}$. Another possibility is the presence of short-range correlations suggested by specific heat measurements where an anomalous broadening of the Schottky peak caused by the Zeeman splitting of the $\Gamma_{7}^{\mathrm{CEF}}$ ground state is observed [12]. Such short-range dynamical correlations were found to coexist with single-site (i.e. q-independent) fluctuations in a number of Kondo systems [13], but the weight of their signals integrated in $q$-space is usually much smaller than the weight of the quasi-elastic contribution. This fact could explain the absence of an anomalous behavior of the neutron line-width measured at a given q-value. Interestingly, the specific heat data indicate that the short-range correlations are detectable only below the superconducting transition, as also suggested by the $\mu \mathrm{SR}$ data, pointing to a possible interplay between the occurrence of the superconducting state and the magnetic correlations.

We thank Prof. H. Sato and Dr. Y. Aoki for fruitful discussions and the help during the sample preparation. This work was partly supported by the European Science Foundation (FERLIN programme). 


\section{References}

[1] N.D. Mathur et al., Nature 394 (1998) 39.

[2] M. Ishikawa et al., in Superconductivity in d- and f-Band Metals, Eds. W. Buckel and W. Weber (Kernforschungszentrum, Karlsruhe, 1982), p. 141.

[3] H.A. Kierstead et al., Phys. Rev. B 34 (1985) 135.

[4] A. Dönni et al., Physica B 259-261 (1999) 705.

[5] S.K. Malik et al., Phys. Rev. B 32 (1985) 4426.

[6] A. Amato, Rev. Modern Phys. 69 (1997) 1119.

[7] P. Dalmas de Réotier et al., J. Phys.: Condens. Matter 8(1996) 5113.

[8] D.L. Cox et al., J. Appl. Phys. 57 (1985) 3166

[9] W.-H. Li et al., Phys. Rev. B 39 (1989) 4119.

[10] S.K. Malik et al., Phys. Rev. B 31 (1985) 6971.

[11] A. Schenck and F.N. Gygax, in Handbook of Magnetic Materials, vol. 9, ed. K.H.J. Buschow (Elsevier, Amsterdam, 1995).

[12] Y. Aoki et al., Physica C 333 (2000) 187.

[13] J. Rossat-Mignod et al., J. Magn. Magn. Mat. 76\&77 (1986) 376. 


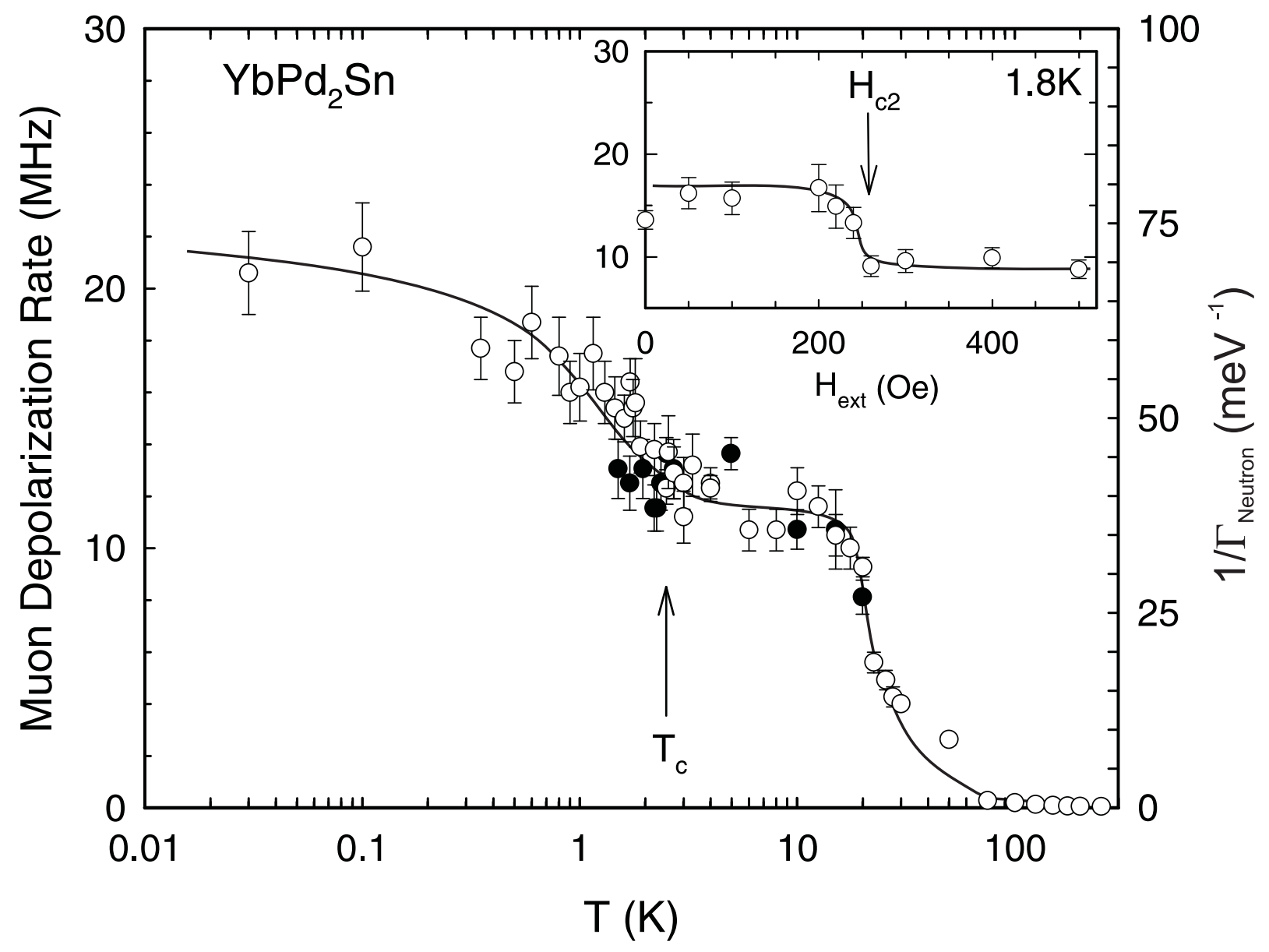

Fig. 1. Temperature dependence of the fast component of the ZF muon depolarization rate (open symbols, left axis). To allow comparison, the inverse of the neutron quasi-elastic linewidth is also reported (closed symbols, right axis). Both quantities are related through Eqs. 3, 4 and 6. The inset shows the field dependence of the muon depolarization rate measured in $\mathrm{LF}$ at $1.8 \mathrm{~K}$ where the data were obtained in a field-cooling procedure. 


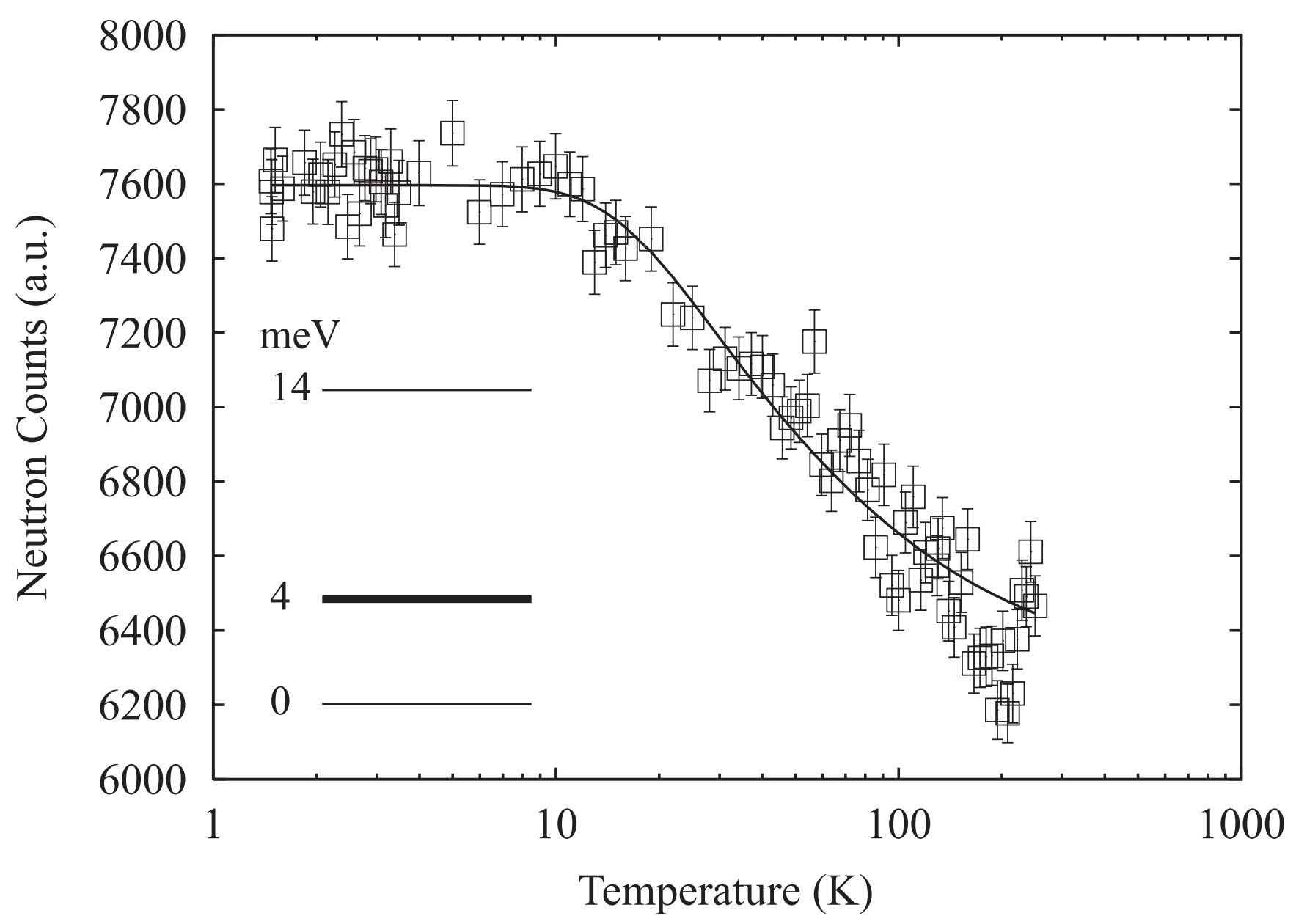

Fig. 2. Temperature dependence of the integrated weight of the quasi-elastic signal obtained at $\mathrm{Q}=0.15 \AA^{-1}$. The line represents the best fit obtained with Eq. 4 . From the fit parameters, the CEF shown in the inset is calculated (ground state $\Gamma_{7}^{\mathrm{CEF}}$ doublet; first excited state $\Gamma_{8}^{\mathrm{CEF}}$ quadruplet; and second excited state $\Gamma_{6}^{\mathrm{CEF}}$ doublet). 